\title{
The Effect of Inoculum Concentration on the Growth Profile and Antibacterial Activity of Actinomycete (A6K) Isolates
}

\author{
Ika Maylani Ningsih and Nanik Sulistyani* \\ Faculty of Pharmacy, Universitas Ahmad Dahlan, Yogyakarta, Indonesia
}

\begin{abstract}
Actinomycetes are important microorganisms renowned for their ability to produce secondary metabolites as antibiotics. A6K Actinomycetes were isolated from papaya plant soil and yielded antibacterial metabolites. This research aims to identify the effect of inoculum concentration on the growth profile and antibacterial activity of A6K isolates as producers of antibiotics. The bacterial concentration within the inoculum (CFU/mL) was determined by a turbidity test using visible spectrophotometry on inoculum $10 \%, 20 \%, 30 \% \mathrm{v} / \mathrm{v}$. The growth profile of A6K isolates was made with a correlation graph between incubation time and weight of biomass in the liquid cultures of A6K isolates, while the temporal profile of secondary metabolites was established with a graph correlating incubation time and growth inhibition zones of $S$. aureus and $E$. coli. Statistical analysis by repeated ANOVA was performed to verify the influence of inoculum concentration rate and incubation period on inhibition zone and biomass weight. According to the growth profile graph, the stationary phase occurred after the fourth day of incubation. Results revealed that inoculum concentration and incubation period gave a significant difference to the resulting inhibition zones. The higher the concentration of inoculum, the larger the bacterial content and the shorter the incubation time needed to obtain maximum antibacterial activity.
\end{abstract}

Keywords: Antibacterial, Actinomycetes, Inoculum Concentration, A6K Isolates.

\section{INTRODUCTION}

The occurrence of resistance due to inappropriate use of antibiotics is more frequent than the discovery of new antibiotics. ${ }^{1}$ It is therefore necessary to seek antibiotics from unprecedented lines with higher potency. A novel antibacterial source under much investigation nowadays comes from Actinomycete bacteria, which produce $70 \%$ of antibiotic compounds. ${ }^{2,3}$ Grass rhizospheres release exudates serving as life source for soil microflora including Actinomycetes, which is why Actinomycetes are often found in grass soil. ${ }^{4,5}$

Arifin ${ }^{6}$ successfully isolated and purified Actinomycetes from the rhizosphere of Carica papaya $L$. of the family Caricaceae, resulting in 15 isolates. Eight of these isolates were proven to inhibit the development of Escherichia coli and Staphylococcus aureus bacteria. One of them, the A6K isolate, had the greatest inhibitive power, generating a radical inhibition zone of $20.75 \mathrm{~mm}$ on the Escherichia coli test bacteria and $24.75 \mathrm{~mm}$ on the Staphylococcus aureus test bacteria. ${ }^{6}$

However, the study in question has yet to disclose how the starter concentration affects the growth profile and antibacterial activity of the eight isolates' liquid cultures. Learning the effect of inoculum concentration in the aforementioned isolates on their

*Author to whom correspondence should be addressed. growth profile and antibacterial activity is essential in determining the suitable time to harvest secondary metabolites, thus simplifying the estimation of the bacteria's incubation period. For this purpose, the isolate tested was the one with the strongest inhibitive power, the A6K isolate.

Actinomycetes grow into mold-like thin filaments to the extent that Actinomycetes are regarded as fungi. On an agar plate, Actinomycetes can be easily distinguished from aerial hyphae and substrate mycelia bacteria. ${ }^{7}$ This study was conducted to identify the effect of inoculum concentration on growth profile and antibacterial activity against Escherichia coli and Staphylococcus aureus. The concentration rate of inoculum impacts on the incubation period length and resulting inhibition zone. The higher the inoculum concentration, the larger the inhibition zone it creates. ${ }^{8,9}$

This research is useful in obtaining the optimal harvest time for antibacterial secondary metabolites in identified inoculum concentration, and thus can be used as basis for future research.

\section{EXPERIMENTAL DETAILS}

\subsection{Material}

The sample utilized in this study was A6K isolated from papaya (Carica papaya) plant soil. ${ }^{6}$ 


\subsection{Actinomycete Isolate Preparation}

As much as $2 \mathrm{~mL}$ active $\mathrm{A} 6 \mathrm{~K}$ isolate in this research was recultured onto a $20 \mathrm{~mL}$ SNB medium, then incubated at room temperature with a stirrer for five days and subjected to streaking (1 ose) in an SNA medium containing $50 \mu \mathrm{g} / \mathrm{mL}$ cycloheximide. ${ }^{6}$ The isolate was subsequently incubated for seven days at room temperature before the growth uniformity of the Actinomycete colony was examined. ${ }^{10}$ The isolate is considered pure if the isolate grows uniformly in line with the morphological traits of an Actinomycete colony. 5,7

\subsection{Characteristics of the A6K Actinomycete Isolate \\ 2.3.1. Morphology}

Morphological testing with observation employed a slide culture method, where the colony from the starter culture was streaked on an SNA medium and a glass cover slip was put on the medium, before being incubated at $28^{\circ} \mathrm{C}$ for a week. Observation on the structure, color and form of the spore used a microscope with $400 \times$ magnification. $^{2}$

\subsubsection{Carbon Source Utilization Test}

An A6K Actinomycete stock culture was streaked on an ISP9 medium, then incubated at room temperature for 10-14 days. ${ }^{2,11}$

\subsection{Starter Culture Preparation}

Five most fertile A6K isolate plugs were inserted into a $250 \mathrm{~mL}$ SNB medium that had been sterilized beforehand. ${ }^{12}$ The culture was thus incubated at room temperature for five days while using a stirrer.

\subsection{Solution Turbidity Testing on Varied Inoculum Concentrations}

This was realized by making a series of McFarland standard concentrations $(\mathrm{CFU} / \mathrm{mL})$ with standard curves of a mixture of $1 \%$ $\mathrm{BaCl}_{2}$ and $1 \% \mathrm{H}_{2} \mathrm{SO}_{4}$ as in Table $\mathrm{I}^{13}$

Absorbance of the McFarland standard solution series was read on a visible spectrophotometer at lambda $600 \mathrm{~nm}$ to make standard curves of bacteria concentration $(\mathrm{CFU} / \mathrm{mL})$ versus absorbance. ${ }^{13}$ Afterwards, the absorbance of each liquid culture $(10,20,30 \% \mathrm{v} / \mathrm{v})$ was read with the same lambda at the first, second, third, fourth and fifth minutes by means of SNB blanks.

Table I. McFarland standard concentrations and composition of their components.

\begin{tabular}{lccc}
\hline & & \multicolumn{2}{c}{ Composition } \\
\cline { 3 - 4 } McFarland standard & $\left(\times 10^{6}\right) \mathrm{CFU} / \mathrm{mL}$ & $1 \% \mathrm{BaCl}_{2}(\mathrm{~mL})$ & $1 \% \mathrm{H}_{2} \mathrm{SO}_{4}(\mathrm{~mL})$ \\
\hline 0.5 & $<300$ & 0.05 & 9.95 \\
1 & 300 & 0.1 & 9.9 \\
2 & 600 & 0.2 & 9.8 \\
3 & 900 & 0.3 & 9.7 \\
4 & 1200 & 0.4 & 9.6 \\
5 & 1500 & 0.5 & 9.5 \\
6 & 1800 & 0.6 & 9.4 \\
7 & 2100 & 0.7 & 9.3 \\
8 & 2400 & 0.8 & 9.2 \\
9 & 2700 & 0.9 & 9.1 \\
10 & 3000 & 1.0 & 9.0 \\
\hline
\end{tabular}

\subsection{A6K Isolate Fermentation}

Stock solutions of $10 \mathrm{~mL}, 20 \mathrm{~mL}$, and $30 \mathrm{~mL}$ respectively were transferred to different new Erlenmeyer flasks containing $100 \mathrm{~mL}$ of new sterilized SNB with three times replication of each concentration. All test cultures were then shaken at $200 \mathrm{rpm}$ and incubated at room temperature for 14 days. During incubation, everyday $1.5 \mathrm{~mL}$ cultures are retrieved and placed in Eppendorf tubes before they were labelled and stored in a freezer. ${ }^{14}$

\subsection{A6K Isolate Supernatant Preparation}

After 14 days, the test cultures were taken out of the freezer and thawed. Once thawed the cultures were centrifuged at $8000 \mathrm{rpm}$ for 10 minutes to separate supernatants from sediments. The obtained supernatants were put into new Eppendorf tubes, designated as test liquid cultures, while the remaining sediments were dried in an oven at $50{ }^{\circ} \mathrm{C}$ until reaching constant weight. $^{15}$

\subsection{Test Bacteria Preparation \\ 2.8.1. Production of Test Bacteria Stock}

The test bacteria used in this research were Staphylococcus aureus and Escherichia coli, prepared by taking 1 ose from each of the utilized pure breed bacteria stocks and placing them into $1 \mathrm{~mL} \mathrm{BHI}$, incubated at $37^{\circ} \mathrm{C}$ for $18-24$ hours. After the bacteria grew, they were stored at $4{ }^{\circ} \mathrm{C}$ as bacteria stock. ${ }^{16,17}$

\subsubsection{Production of Test Bacterial Suspension}

One ose from the bacteria stock was suspended in a tube and received $1 \mathrm{~mL}$ BHI before being incubated for 18-24 hours at $37{ }^{\circ} \mathrm{C}$. $100 \mu \mathrm{L}$ was collected from the incubation, immersed in $1 \mathrm{~mL}$ BHI and incubated further for 3-5 hours at $37{ }^{\circ} \mathrm{C}$. Afterwards, $100 \mu \mathrm{l}$ was retrieved and added with $\mathrm{NaCl} 0.9 \%$ until its turbidity matched the McFarland standard $\left(10^{8} \mathrm{CFU} / \mathrm{mL}\right) .{ }^{16}$

\subsection{Test of the Activity of A6K Actinomycete Isolate Liquid Culture Against Test Bacteria}

The test bacteria for the A6K isolate liquid culture activity test were Escherichia coli and Staphylococcus aureus, while the applied medium was a Mueller Hinton (MH) medium. A Petri dish with the MH medium was smeared with the test bacterial suspension using a sterile cotton swab by dipping it into the suspension and squeezing it against the tube wall, then smearing the suspension evenly on the MH agar medium surface and leaving it for \pm 30 minutes until it is absorbed. Every $20 \mu \mathrm{L}$ test liquid culture from the $\mathrm{A} 6 \mathrm{~K}$ isolate was dropped onto a paper disc $6 \mathrm{~mm}$ in diameter according to day order (1-14) and incubated for $18-24$ hours at $37{ }^{\circ} \mathrm{C} .^{2}$

After 24 hours, observation was conducted on the antibacterial activity of the A6K isolate test liquid culture, which is marked by a clear zone around the paper disc. Next, the inhibition diameter was estimated by measuring the diameter of the clear zone around the paper disc from four sides of the inhibition zone circle and calculating the average diameter. ${ }^{18}$ The acquired data were then presented in a graph between incubation period (day) and inhibition zone diameter $(\mathrm{mm}) .{ }^{16}$ Statistical analysis was also run to find out whether there was a significant difference in the effect of inoculum concentration on the inhibition zone. 


\subsection{Test of the Effect of Inoculum Concentration on the Growth Profile of the A6K Actinomycete Isolate}

The growth profile of the A6K isolates from day one to 14 was obtained by examining the weight of sediments resulting from incubation day 1-14. Each sediment separated from its supernatant in an oven at $50{ }^{\circ} \mathrm{C}$ was weighed. Dry sediments were weighed until achieving constant weight and a graph between incubation time (day) and biomass ( $\mathrm{mg}$ ) was made. In addition, statistical analysis was performed to identify any meaningful difference in the impact of inoculum concentration on the growth of the A6K isolates.

\section{RESULTS AND DISCUSSION}

When planted in an SNA medium, the A6K isolate (Fig. 1) showed characteristics of a slow-developing colony, in that it took two days before starting to grow. Following the fourth day, the colony's yellow pigments appeared, adhered strongly to the agar, and was damp (not dry). The colony grew slowly and had a uniform color, indicating no growth of fungi or other bacteria. The colony developing in the SNA medium underwent microscopic examination by being Gram stained beforehand. ${ }^{19}$ The microscopic test outcome revealed that the colony growing in the SNA medium was that of the A6K Actinomycete isolate. This finding was similar to microscopic test results in prior research, ${ }^{6}$ was purple in color (Gram-positive bacteria), and assumed the shape of branched filaments or had mycelia.

Once microscopic test results confirmed that the cultured bacteria were indeed $\mathrm{A} 6 \mathrm{~K}$ Actinoycetes, the next step was to create a starter culture in an SNB medium and a carbon test in Nitrate Agar medium by ISP9 procedure. $^{2}$ The application of carbon sources was meant to determine in which carbon source the Actinomycete bacteria could develop fast and productively. This carbon source test undertook a procedure akin to that of ISP9. ${ }^{2,11}$ This study employed carbon sources from sucrose, glucose, mannitol, fructose and rhamnose, and used SNA as positive control as delineated in Table II.

The outcomes of carbon source usage showed that the fastest growth in comparison with the positive control was in Nitrate Agar medium with glucose monohydrate on the second day, while the slowest was in Nitrate Agar medium with mannitol where the development only intensified on the fourth day, aside from being less prolific than the negative control.

Subsequently, the obtained starter cultures were each inoculated in $100 \mathrm{~mL} \mathrm{SNB}$ in a $250 \mathrm{~mL}$ Erlenmeyer flask by varying the concentrations at $10,20,30 \% \mathrm{v} / \mathrm{v}$ with threefold replication of

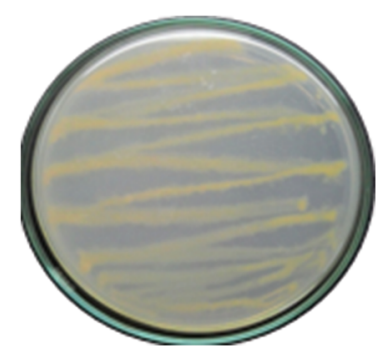

Fig. 1. A6K Actinomycete isolate in SNA medium.
Table II. A6K Actinomycete isolate growth in certain carbon sources.

\begin{tabular}{cccccccc}
\hline \multicolumn{7}{c}{ Growth } \\
\cline { 2 - 8 } Day & $\begin{array}{c}\text { Negative } \\
\text { control }\end{array}$ & $\begin{array}{c}\text { Positive } \\
\text { control }\end{array}$ & Mannitol & Sucrose & Lactose & Rhamnose & Glucose \\
\hline 0 & - & - & - & - & - & - & - \\
1 & + & + & + & + & + & + & + \\
2 & + & + & + & + & + & + & ++ \\
3 & + & +++ & + & ++ & ++ & ++ & +++ \\
4 & + & +++ & ++ & +++ & ++ & +++ & +++ \\
5 & ++ & +++ & ++ & +++ & ++ & +++ & +++ \\
6 & ++ & +++ & ++ & +++ & ++ & +++ & +++ \\
7 & ++ & +++ & ++ & +++ & ++ & +++ & +++ \\
\hline
\end{tabular}

each concentration before undergoing the turbidity test. ${ }^{14}$ The linear regressive equation between McFarland standard concentration and absorbance gained in this study was $y=0.00052 x+$ 0.06053 with $R=0.99607$.

The bacteria concentration prior to incubation calculated through that equation was $300.264 \mathrm{CFU} / \mathrm{mL}$ in inoculum $10 \% \mathrm{v} / \mathrm{v}, 1,608.596 \mathrm{CFU} / \mathrm{mL}$ in inoculum $20 \% \mathrm{v} / \mathrm{v}$, and $1,699.622 \mathrm{CFU} / \mathrm{mL}$ in inoculum $30 \% \mathrm{v} / \mathrm{v}$, whereas inoculum $100 \% \mathrm{v} / \mathrm{v}$ contained $1,906.673 \mathrm{CFU} / \mathrm{mL}$ of bacteria. This finding implies that the larger the percentage of inoculum tested, the greater its bacterial content. Following 14 days of incubation, the growth curve of each inoculum concentration was yielded as displayed in Figure 2.

The bacteria growth curve can be established by determining its dry weight, which is one of the best methods of calculation for Actinomycetes. ${ }^{18}$ Actinomycete growth can be examined by measuring the biomass weight of the tested liquid culture and then depicting it in a curve correlating incubation period (day) to $\log$ biomass (mg) to identify the growth profile of the A6K Actinomycete isolate starter.

It can be seen from Figure 2 that at inoculum concentrations of $20 \% \mathrm{v} / \mathrm{v}$ and $30 \% \mathrm{v} / \mathrm{v}$ the exponential phase peaked or attained optimal bacteria growth on the fourth day and thereafter declined, denoting the onset of the stationary phase or even death. At inoculum concentration of $10 \% \mathrm{v} / \mathrm{v}$ the exponential phase peaked on the third day and then also entered the stationary phase until day 6 . When the bacteria come into

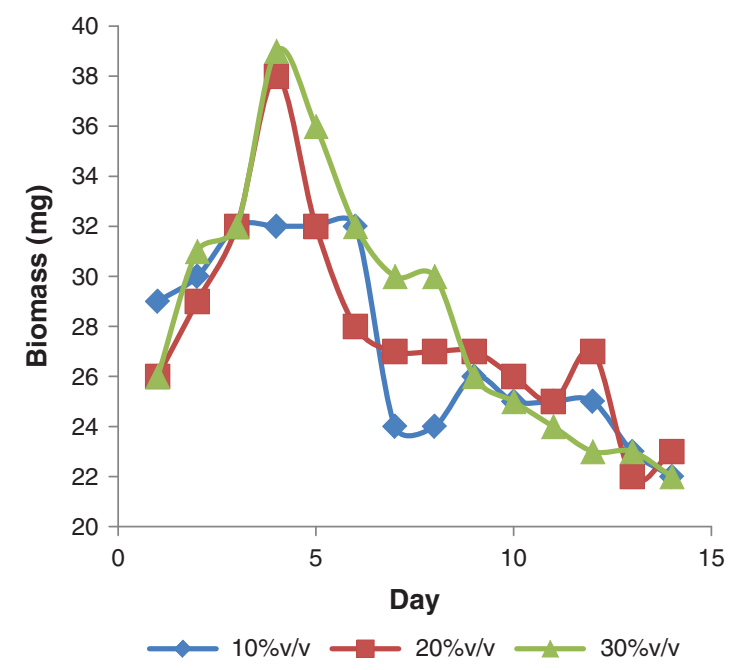

Fig. 2. A6K isolate growth curve. 
Table III. A6K antibacterial activity against Escherichia coli.

\begin{tabular}{lccc}
\hline & \multicolumn{3}{c}{ Inhibition zone diameter $(\mathrm{mm})$} \\
\cline { 2 - 4 } Days & $10 \% \mathrm{v} / \mathrm{v}$ & $20 \% \mathrm{v} / \mathrm{v}$ & $30 \% \mathrm{v} / \mathrm{v}$ \\
\hline 6 & $3.55 \pm 0.10$ & $6.56 \pm 1.10$ & $10.03 \pm 0.14$ \\
7 & $5.16 \pm 0.88$ & $6.34 \pm 0.08$ & $10.66 \pm 0.43$ \\
8 & $6.64 \pm 0.37$ & $3.49 \pm 0.03$ & $6.50 \pm 1.84$ \\
9 & $2.57 \pm 0.27$ & - & $3.21 \pm 0.03$ \\
10 & $1.22 \pm 0.01$ & - & - \\
\hline
\end{tabular}

the stationary phase after passing the exponential peak, they begin producing secondary metabolites. The stationary phase is prompted by depleting nutrients, $\mathrm{pH}$ change, and the same number of dead and new cells so that the bacteria compete to survive by excreting self-protective substances (secondary metabolites), among which are antibacterial. ${ }^{19}$

The resemblance in growth profile in inoculum $20 \% \mathrm{v} / \mathrm{v}$ and $30 \% \mathrm{v} / \mathrm{v}$ was probably caused by similar bacterial content prior to incubation. The $10 \% \mathrm{v} / \mathrm{v}$ concentration generated a stationary phase which lasted for four days (days 3-7). This suggests that an inoculum concentration of $10 \% \mathrm{v} / \mathrm{v}$ is better for harvesting during the stationary phase to gain antibiotics than $20 \% \mathrm{v} / \mathrm{v}$ and $30 \% \mathrm{v} / \mathrm{v}$ because both reached the death phase sooner. However, statistical assessment on data from day 1 to 14 generally showed no significant difference in growth profile among the three inoculum concentrations.

The supernatants of the A6K isolate liquid cultures from day 1 until 14 were then tested for antibacterial activity against Staphylococcus aureus and Escherichia coli. Results of the antibacterial activity test are summarized in Table III (E. coli) and Table IV (S. aureus). In the testing on E. coli, antibacterial activity was initially detected on the sixth day with the shortest inhibition zone diameter at inoculum $10 \% \mathrm{v} / \mathrm{v}(3.55 \mathrm{~mm})$, followed by that at $20 \% \mathrm{v} / \mathrm{v}(6.56 \mathrm{~mm})$ and the longest at inoculum $30 \% \mathrm{v} / \mathrm{v}$ $(10.03 \mathrm{~mm})$. This signifies that on day 6 most active metabolites were procured in inoculum $30 \%$. A similar occurrence transpired in the test on S. Aureus, which disclosed that on the sixth day antibacterial activity only began to manifest in inoculum $30 \% \mathrm{v} / \mathrm{v}$, whilst the other inoculum concentrations have yet to reveal such activity. It can therefore be concluded that the greater the inoculum concentration, the faster active antibacterial metabolites are engendered.

According to outcomes of the test on E. coli, optimal antibacterial activity was provided on day 8 (in inoculum $10 \% \mathrm{v} / \mathrm{v}$ ), day 6 or day 7 (in inoculum 20\% v/v and 30\% v/v respectively). Maximum inhibition zone diameter was yielded by inoculum $30 \%$ v/v. Meanwhile, in results of the test on S. aureus, optimal antibacterial activity was produced on the ninth day (in inoculum $10 \% \mathrm{v} / \mathrm{v}$ ), eighth day (in inoculum $20 \% \mathrm{v} / \mathrm{v}$ ) and seventh day (in inoculum 30\% v/v). Maximum inhibition zone diameter was resulted by inoculum $10 \%$ v/v. Maximum activity generated by the three concentrations against the test bacteria was first achieved by inoculum $30 \% \mathrm{v} / \mathrm{v}$, followed by $20 \% \mathrm{v} / \mathrm{v}$ and lastly $10 \% \mathrm{v} / \mathrm{v}$. This indicates that the higher the concentration, the faster maximum antibacterial activity is attained. Meanwhile, the optimal incubation time of each inoculum concentration in the tests on E. coli and $S$. aureus were virtually the same, raising the assumption that the metabolites which actively subjugated E. coli and $S$. aureus were identical.
Table IV. A6K antibacterial activity against Staphylococcus aureus.

\begin{tabular}{lccc}
\hline & \multicolumn{3}{c}{ Inhibition zone diameter $(\mathrm{mm})$} \\
\cline { 2 - 4 } Day & $10 \% \mathrm{v} / \mathrm{v}$ & $20 \% \mathrm{v} / \mathrm{v}$ & $30 \% \mathrm{v} / \mathrm{v}$ \\
\hline & - & - & $4.03 \pm 0.18$ \\
7 & $3.90 \pm 0.04$ & $4.10 \pm 0.81$ & $9.80 \pm 0.24$ \\
8 & $6.18 \pm 1.31$ & $9.63 \pm 0.21$ & - \\
9 & $10.77 \pm 0.52$ & - & - \\
10 & $1.22 \pm 0.01$ & - & - \\
\hline
\end{tabular}

Overall, statistical test results showed a significant difference stemming from the influence of inoculum concentration and incubation time on the yielded inhibition zone against $E$. coli. Likewise, statistical analysis also revealed a meaningful difference resulting from the effect of inoculum concentration and incubation time on the inhibition zone generated around S. aureus.

\section{CONCLUSIONS}

The growth profile indicated that the stationary phase commenced after the fourth day, but statistical analysis results suggested no impact of inoculum concentration on the biomass weight produced, since there was no significant difference either based on variation in concentration or incubation period length.

Nevertheless, inoculum concentration rate does affect the antibacterial activity profile of the A6K Actinomycete isolate. The higher the inoculum concentration, the larger the bacterial content and the shorter the incubation time required to obtain optimal inhibition zone.

Incubation time does not exert any effect on biomass weight but does impact on the inhibition zone created by the A6K Actinomycete isolate.

\section{References and Notes}

1. R. Bisth, A. Katiyar, R. Singh, and P. Mittal, Asian J. Pharm. Clin. Res. 2, 2 (2009).

2. H. Rante, Wahyono., Y. B. Murti, and G. Alam, Indonesian J. Pharm. 21, 3 (2010).

3. S. J. Kumar, P. Santhanam, and M. M. Selvam, Int. J. Pharma. Bio. Sci. 2, 1 (2011).

4. S. A. Waksman, The Actinomycetes (1950)

5. M. Good Fellow, S. T. William, and M. Moordarski, Actinomycetes in Biotechnol. 188, 327 (1988).

6. M. Arifin, Thesis (2015).

7. S. Qin, J. Li, H. H. Chen, G. Z. Zhao, W. Y. Zhu, C. L Jiang, L. H. Xu, and W. J. Li, Appl. Environ. Microbiol. 75 (2009).

8. S. Febina, R. D. Rachel, and R. Shenbagaratai, Int. J. Pharm. Pharm. Sci. 2, 6 (2014).

9. M. A. Houssam, A. S. El-Sayed, H. Mona, and M. El-Gazar, World Rural Observations 3, 3 (2011).

10. Y. Jiang, Q. Li, X. Chen, and C. Jiang, Isolation and Cultivation Methods of Actinobacteria 42 (2016).

11. E. B. Shirling and D. Gottlieb, Intr. J. Syst. Bact. 13 (1996).

12. X. Wang, L. Huang, Z. Kang, H. Buchenauer, and X. Gao, J. Biomedicine Biotechnol. 10 (2010).

13. S. Sutton, J. Validation Technol. (2011).

14. N. Gaurav, S. Shukla, R. Mall, and K. Mishra, Eur. J. Biomedical and Pharm. Sci. 2, 4 (2015).

15. M. Oskay, Int. J. Agric. Biol. 13, 3 (2011).

16. N. Sulistyani and Mulyadi, J. Public Health 7, 2 (2013).

17. M. Oskay, A. U. Tamer, and C. C. Azeri, Afr. J. Biotechnol. 3, 9 (2004).

18. V. Parkavi, M. Vignesh, K. Selvakumar, J. M. Mohamed, and J. J. Roby, Int. J. Pharm. Sci. Drug Res. 4, 3 (2012).

19. G. Sumbali and R. S. Mehrotra, Principles Microbiol. 94 (2009, 2010). 